\title{
Context encoding enables machine learning-based quantitative photoacoustics (Erratum)
}

\author{
Thomas Kirchner \\ Janek Gröhl \\ Lena Maier-Hein
}




\title{
Context encoding enables machine learning-based quantitative photoacoustics (Erratum)
}

\author{
Thomas Kirchner, ${ }^{\mathrm{a}, \mathrm{b}}$ Janek Gröhl, ${ }^{\mathrm{a}, \mathrm{c}}$ and Lena Maier-Hein ${ }^{\mathrm{a}, \mathrm{c}}$ \\ ${ }^{a}$ German Cancer Research Center (DKFZ), Division of Computer Assisted Medical \\ Interventions (CAMI), Heidelberg, Germany \\ ${ }^{b}$ Heidelberg University, Faculty of Physics and Astronomy, Heidelberg, Germany \\ ${ }^{c}$ Heidelberg University, Medical Faculty, Heidelberg, Germany
}

[DOI: 10.1117/1.JBO.25.4.049802]

This article [J. Biomed. Opt. 23(5), 056008 (2018) doi: 10.1117/1.JBO.23.5.056008 was originally published online on 18 May 2018 with an error in the reported number for the reduced scattering coefficient. The authors reported a reduced scattering coefficient of $15 \mathrm{~cm}^{-1}$; however, this number was not the reduced scattering coefficient, but the normal scattering coefficient.

To correct the error, the reported number for the reduced scattering coefficient has been adjusted from $15 \mathrm{~cm}^{-1}$ to $1.5 \mathrm{~cm}^{-1}$ in three instances in the article:

1. Section 2.1, Fluence Contribution Map, second paragraph;

2. Section 3.1.1, Experiment, second paragraph; and

3. Section 3.2.1, Experiment, first paragraph.

In the article, the authors claim the general feasibility of the proposed method towards fluence estimation from photoacoustic initial pressure data. By the opinion of all involved authors, this claim is not impeded by the reporting error.

This article was corrected online on 7 April 2020. 